\title{
Functional response to inhaled salbutamol and/or ipratropium bromide in Ascaris suum-sensitised cats with allergen-induced bronchospasms
}

\author{
Jérôme Leemans $^{\mathrm{a}, *}$, Nathalie Kirschvink ${ }^{\mathrm{c}}$, Cécile Clercx $^{\mathrm{b}}$, Carole Cambier ${ }^{\mathrm{a}}$, Pascal Gustin ${ }^{\mathrm{a}}$ \\ a Section of Pharmacology, Pharmacotherapy and Toxicology, Department for Functional Sciences B41, Faculty of Veterinary Medicine, University of Liège, 4000 Liège, Belgium \\ ${ }^{\mathrm{b}}$ Department for Clinical Sciences B44, Faculty of Veterinary Medicine, University of Liège, 4000 Liège, Belgium \\ ${ }^{\mathrm{c}}$ Animal Physiology, Department for Veterinary Medicine, Faculty of Sciences, University of Namur, 5000 Namur, Belgium
}

\section{A R T I C L E I N F O}

\section{Article history:}

Accepted 25 July 2009

\section{Keywords:}

Feline

Early asthmatic reaction

Barometric whole-body plethysmography

Inhaled bronchodilators

\begin{abstract}
A B S T R A C T
Knowledge about the use of inhaled bronchodilators in cats with so-called 'feline asthma' is limited and relies on the experience of clinicians treating these patients. A randomised controlled four-way crossover study was therefore designed to compare the effects of salbutamol (SAL, $100 \mu \mathrm{g})$, ipratropium bromide $(\mathrm{IB}, 20 \mu \mathrm{g})$ and a combination of both $(\mathrm{SAL} / \mathrm{IB}, 100 \mu \mathrm{g} / 20 \mu \mathrm{g})$, delivered through a pressurised metereddose inhaler (pMDI) connected to a spacing chamber, on allergen-induced bronchospasms in five Ascaris suum (AS)-sensitised cats. Four AS bronchial provocation challenges were carried out at 1 week intervals, followed by one of four treatment protocols: SAL, IB, SAL/IB or control (untreated). Enhanced pause (Penh), an estimator of airflow limitation measured by barometric whole-body plethysmography, was repeatedly assessed within $120 \mathrm{~min}$ following the administration of each treatment protocol. Responses to inhaled medications were evaluated by calculating the area under the time-response curves (AUC) from 0 to 60 or 120 min after drug administration $\left(\mathrm{AUC}_{0-60}, \mathrm{AUC}_{0-120}\right)$, as well as the times required for half-recovery $\left(T_{50 \%}\right)$ or for returning to nearly basal conditions $\left(T_{20 \%}\right)$.

No significant differences were found among the four study groups, with reference to the mean $\mathrm{AUC}_{0-}$ ${ }_{60}, T_{20 \%}$ and $T_{50 \%}$ values of Penh $(P>0.05)$. Mean $A_{U C} C_{0-120}$ values of Penh were similar between the bronchodilators tested, but were significantly lower than those in the untreated group. It was concluded that inhalation of SAL, IB and SAL/IB via pMDI failed to improve most Penh-derived parameters, which suggested that these bronchodilators were of limited efficacy in reversing allergen-induced bronchospasm in cats. However, further studies using a larger number of animals are warranted to investigate if different drugs or delivery devices or higher dosages may be more effective.
\end{abstract}

(c) 2009 Elsevier Ltd. All rights reserved.

\section{Introduction}

Feline allergic asthma is a chronic inflammatory disorder within the lower respiratory tract that is commonly encountered in pet cats. Clinical signs include chronic coughing, wheezing and intermittent respiratory distress. These symptoms arise from obstruction of the lower airways caused by airway wall oedema, excessive mucus secretion, inflammatory cellular infiltrates and reversible airway smooth-muscle constriction in response to specific aeroallergens (Padrid, 2000; Hirt, 2005). Non-allergic stimuli (dust litter, smoke or stress) have been hypothesised to trigger bronchoconstriction in a non-specific manner. Because the clinical signs observed in feline asthma are at least partly due to reversible airway smooth-muscle constriction, bronchodilator therapy should be effective for symptomatic relief of acute respiratory distress.

\footnotetext{
* Corresponding author. Tel.: +32 436642 58; fax: +32 43664176

E-mail address: J.Leemans@student.ulg.ac.be (J. Leemans).
}

Because cats spontaneously show allergic asthma, particular emphasis has been placed on developing feline models of allergic asthma to further explore the pathogenesis of the human condition. On the basis of sensitisation to experimental (Ascaris suum) or clinically relevant allergens (Bermuda grass, house-dust mite), these models successfully mimicked many hallmark features of both feline and human diseases: eosinophilic airway inflammation, reversible airflow limitation subsequent to inhalation of specific allergens, non-specific bronchial hyperresponsiveness and airway remodelling (permanent structural changes within airways) at advanced stages (Padrid et al., 1995; Norris Reinero et al., 2004; Kirschvink et al., 2007).

More interestingly, biphasic increases in lung resistance were evidenced in A. suum (AS)-sensitised cats, with the following profile: one peak within $10 \mathrm{~min}$ following allergen exposure, a subsequent return to baseline in most cats, and a second peak after $24 \mathrm{~h}$ (Padrid et al., 1995). Although any extrapolation between species should be considered with caution, this peculiar pattern of changes in pulmonary function strikingly evokes the allergen-induced dual 
asthmatic reaction, similar to that reported in a subset of human asthmatics. The early asthmatic reaction (EAR) to inhaled allergen results from IgE-mediated degranulation of mast cells, which release bronchospastic mediators (histamine, eicosanoids), the late asthmatic reaction (LAR) involves the recruitment and activation of eosinophils, $\mathrm{CD}^{+} \mathrm{T}$ cells, basophils, neutrophils and macrophages (Bousquet et al., 2000). However, to the best of our knowledge, no study has been specifically designed to assess the reproducibility of asthmatic reactions following successive allergen challenges, which is critical for the accurate screening of bronchodilating drugs.

Development and implementation of feline asthma models with standardised allergen exposures (type, duration, quantity and timing) has greatly facilitated the search for targeting novel therapies in cats and, to a lesser extent, in human beings. Historically, cyclosporine A, an effective inhibitor of T-cell activation, has been the first drug to be shown to prevent bronchial hyperreactivity and cytological/morphological alterations in the airways of AS-sensitised and chronically challenged cats (Padrid et al., 1996). Potent immunosuppressive effects, requirement for continued monitoring and exorbitant expenses have precluded cyclosporine A from the routine management of feline asthma and emphasise the need to widen therapeutic options. In two randomised controlled trials on cats with experimental asthma, inhaled corticosteroids, either as monotherapy or in combination with long-acting $\beta_{2}$-adrenergic agonists, were reported to display approximately similar efficacy than oral corticosteroids in reducing eosinophilic airway inflammation, thereby representing a valuable alternative for the clinical condition (Reinero et al., 2005; Leemans et al., 2008). Treatments directed towards specific effector systems are unsatisfactory because cyproheptadine (a serotonin antagonist) at low or high doses, cetirizine (a 2nd-generation selective histamine-receptor antagonist) and zafirlukast (cysteinyl leukotriene-1-receptor antagonist) failed to dampen eosinophilic airway inflammation in experimentally asthmatic cats (Reinero et al., 2005; Schooley et al., 2007). In contrast, allergen-specific immunotherapy, which is a curative desensitising therapy for allergic diseases, increasingly promises to be a new approach for feline asthma, although it remains experimental at this stage (Reinero et al., 2006, 2008; LeeFowler et al., 2009). Considering the above concerns, randomised controlled studies that assess the palliative use of bronchodilators in experimentally asthmatic cats with acute airway obstruction are still lacking.

Aerosol therapy offers the advantages of achieving higher concentrations of medications in target tissues while simultaneously attenuating their systemic toxicity (Boothe, 2004). In feline medicine, pulmonary drug delivery may be accomplished either by nebulisers or a human pressurised metered-dose inhaler (pMDI), adapted to animal use by connecting a spacing chamber and a facemask. Recently, a pilot study conducted on healthy cats with carbachol-induced bronchoconstriction addressed both magnitude and duration of the antispasmodic effects of $\beta_{2}$-sympathomimetic and anticholinergic agents administered by pMDI and/or nebulisation (Leemans et al., 2009). It is worthwhile to mention that both methods of delivery lead to a similar degree of bronchoprotection at the doses studied. Moreover, concurrent exposure to salbutamol (SAL) ( $\beta_{2}$-agonist) and ipratropium bromide (IB) (anticholinergic agent) delivered by pMDI was found to generate a highly synergistic interaction. At unknown doses and with unspecified onsets of action, SAL administered through pMDI appeared to reverse airway obstruction in cats diagnosed with asthma (Rozanski and Hoffman, 1999). Although preliminary data suggested that these drugs may be useful for at-home management of asthmatic cats, bronchodilators delivered by pMDI should be studied more closely, especially with reference to anticholinergic drugs, drug interactions, dosages and temporal effects on lung function.
Methods for evaluating the response of bronchodilators over time in cats with allergen-induced EAR include the follow-up of airflow limitation by pulmonary function testing. Barometric whole-body plethysmography (BWBP) has been validated as a non-invasive pulmonary function test for repeated assessments of the respiratory system in un-sedated, unrestrained and spontaneously breathing cats (Kirschvink et al., 2006). Through calculation of the enhanced pause (Penh), a unitless index of airflow limitation correlated with pulmonary resistance (Hamelmann et al., 1997; Halloy et al., 2004; Kirschvink et al., 2005; Hirt et al., 2008), BWBP has allowed the quantification of both airway obstruction and its reversal with a $\beta_{2}$-agonist in cats with naturally developing asthma (Rozanski and Hoffman, 1999).

Using BWBP, the aims of this study were as follows: first, to test the reproducibility of allergen-induced EAR in high-responsive cats sensitised to AS; second, to compare, on the basis of a randomised controlled four-way crossover trial, the effects of SAL (100 $\mu \mathrm{g})$, IB $(20 \mu \mathrm{g})$ and a combination of both (SAL/IB: $100 \mu \mathrm{g} / 20 \mu \mathrm{g})$, delivered by pMDI, on allergen-induced bronchospasms in AS-sensitised cats. The authors hypothesised that inhaled SAL and IB would be effective as monotherapies in reversing early allergenelicited bronchospasm and that combining them would enhance their efficacy in a synergistic fashion.

\section{Material and methods}

Animals

Eight shorthaired AS-sensitised cats, aged 41 months (Harlan Netherlands; four neutered males, four neutered females; mean bodyweight [BW]: $4.7 \pm 0.3 \mathrm{~kg}$ ), were recruited from a colony of cats experimentally sensitised to AS (Kirschvink et al., 2007). The selected cats were between 11 and 29 months of age at initial sensitisation, had a range of 3-8 AS-challenges in previous studies, and all had their last ASchallenge 9 months previously. They had no history of respiratory tract infection within a period of 6 weeks before being entered in the study. To be eligible, the cats had to demonstrate at least two of the following features of the asthma phenotype after AS stimulation: an increase in total cellular count of the bronchoalveolar lavage fluid (BALF) to $>300$ cells $/ \mu \mathrm{L}$; an increase in percentage of BALF eosinophils to $>18 \%$ (Padrid et al., 1991; Hawkins et al., 1994) and/or demonstrable bronchial hyperreactivity to carbachol (defined as a decrease in C-Penh $\mathrm{h}_{300}$ after AS-challenge compared with saline-challenge) (Table 1 ). These data were retrieved from previous research conducted by the same investigators to maximally respect the welfare of the tested animals.

The enrolled animals were housed and cared for according to the national guidelines and the principles proposed by the European Council for the Care of Laboratory Animals. Each cat was weighed at weekly intervals.

\section{Study design}

First, airway responsiveness towards inhaled carbachol, which is a non-invasive indicator of lower airway inflammation and bronchial responsiveness to non-allergic stimuli, was assessed in the selected cats by BWBP (Leemans et al., 2009). Subsequently, the subjects had to successfully complete a 2 week initial inhalation challenge-test period before enrolment in a therapeutic trial based on a randomised controlled four-way crossover design (Fig. 1).

The initial inhalation challenge-test period was carefully designed (1) to screen for an AS-induced EAR among the eight selected subjects and (2) to test the repeatability of AS-related changes in Penh after two consecutive allergen exposures. The screening period consisted of three successive inhalation challenges as follows: sterile saline $(0.9 \% \mathrm{NaCl})$ (Pre-challenge) and saline solutions containing incremental concentrations of AS allergen (Greer Laboratories) (Challenges 1 and 2), respectively, conducted at 1 week intervals. At each time point, the cats underwent repeated pulmonary function tests performed by BWBP. Of the eight subjects screened, five (Table 1; Nos. 3, 4, 6, 7 and 8) fulfilled the eligibility criteria (refer 'Initial inhalation challenge-test period') for entry into the following crossover trial.

The therapeutic trial started at the end of a 2-week washout period. This was a randomised, controlled, single-dose, four-way crossover study aimed at investigating the effects of inhaled bronchodilators delivered by pMDI. On four different study days, the enrolled cats were exposed to the AS allergen. Shortly after the initiation of allergen-induced EAR, they randomly received inhaled SAL (100 $\mu \mathrm{g}, 1$ puff); inhaled IB $(20 \mu \mathrm{g}, 1$ puff); a randomised combination of SAL/IB $(100 \mu \mathrm{g} /$ $20 \mu \mathrm{g}$, each 1 puff); or no drug. The cats were crossed over to the other treatments at 1 week intervals, so that each cat received all four treatments during the study. 
Table 1

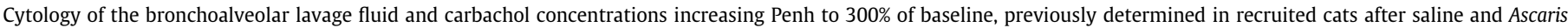
suum challenges.

\begin{tabular}{|c|c|c|c|c|c|}
\hline Cat & Gender & Time points & Total cells (cells/ $\mu \mathrm{L}$ BALF) & Eosinophils (\%) & C-Penh $300 \%(\mathrm{~g} / 100 \mathrm{~mL})$ \\
\hline 1 & M & $\begin{array}{l}\text { Saline } \\
\text { A. suum }\end{array}$ & $\begin{array}{l}165 \\
340\end{array}$ & $\begin{array}{l}7 \\
60\end{array}$ & $\begin{array}{l}0.017 \\
0.010\end{array}$ \\
\hline 2 & $\mathrm{~F}$ & $\begin{array}{l}\text { Saline } \\
\text { A. suum }\end{array}$ & $\begin{array}{l}205 \\
316\end{array}$ & $\begin{array}{l}1.5 \\
51.5\end{array}$ & $\begin{array}{l}0.031 \\
0.018\end{array}$ \\
\hline 3 & $\mathrm{~F}$ & $\begin{array}{l}\text { Saline } \\
\text { A. suum }\end{array}$ & $\begin{array}{l}260 \\
736\end{array}$ & $\begin{array}{l}2.5 \\
60\end{array}$ & $\begin{array}{l}0.035 \\
0.045\end{array}$ \\
\hline 4 & $\mathrm{~F}$ & $\begin{array}{l}\text { Saline } \\
\text { A. suum }\end{array}$ & $\begin{array}{l}245 \\
457\end{array}$ & $\begin{array}{l}13 \\
46\end{array}$ & $\begin{array}{l}0.050 \\
0.044\end{array}$ \\
\hline 5 & $\mathrm{M}$ & $\begin{array}{l}\text { Saline } \\
\text { A. suum }\end{array}$ & $\begin{array}{l}67.5 \\
336\end{array}$ & $\begin{array}{l}2 \\
68\end{array}$ & $\begin{array}{l}0.010 \\
0.009\end{array}$ \\
\hline 6 & $\mathrm{M}$ & $\begin{array}{l}\text { Saline } \\
\text { A. suum }\end{array}$ & $\begin{array}{l}109 \\
84\end{array}$ & $\begin{array}{l}5 \\
67\end{array}$ & $\begin{array}{l}0.021 \\
0.011\end{array}$ \\
\hline 7 & $\mathrm{~F}$ & $\begin{array}{l}\text { Saline } \\
\text { A. suum }\end{array}$ & $\begin{array}{l}92.5 \\
637\end{array}$ & $\begin{array}{l}5 \\
18.5\end{array}$ & $\begin{array}{l}0.020 \\
0.019\end{array}$ \\
\hline 8 & M & $\begin{array}{l}\text { Saline } \\
\text { A. suum }\end{array}$ & $\begin{array}{l}117 \\
109\end{array}$ & $\begin{array}{l}7 \\
55\end{array}$ & $\begin{array}{l}0.035 \\
0.018\end{array}$ \\
\hline
\end{tabular}

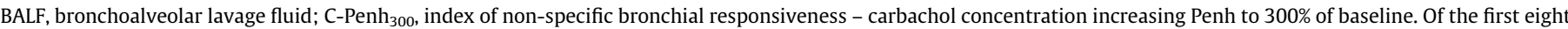
recruited cats, five (Nos. 3, 4, 6, 7 and 8) fulfilled the eligibility criteria for entrance into the comparative bronchodilator trial.

$n=8 \quad A . S$. -sensitised cats

INITIAL INHALATION CHALLENGE TEST PERIOD

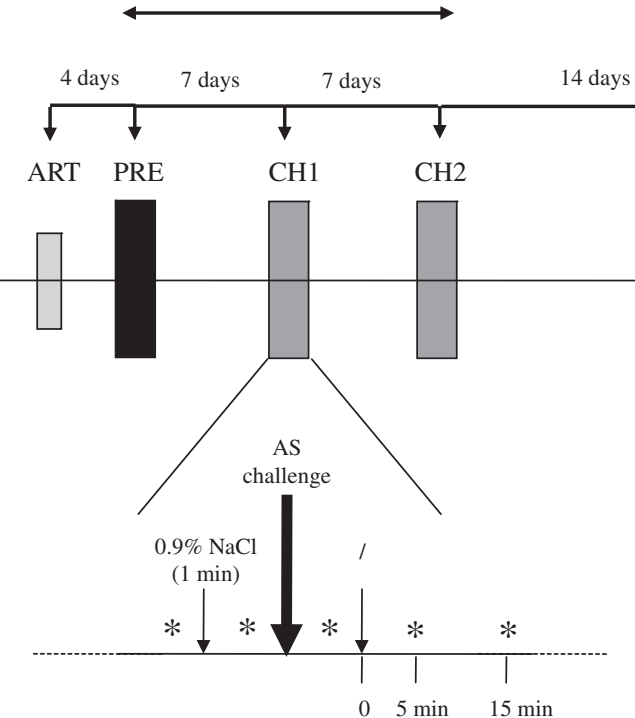

* 5-min session of BWBP

$$
n=5
$$

RANDOMISED, CROSS-OVER, FOUR-WAY, CONTROLLED STUDY : SALBUTAMOL, IPRATROPIUM BROMIDE, COMBINATION, / Liège.

The study was approved by the Animal Ethical Committee of the University of

Initial inhalation challenge-test period

Two baseline recordings of the respiratory variables were carried out on cats both at rest and after a 1 min saline nebulisation with a nebuliser (Ultraneb Devilbiss 2000, Devilbiss Healthcare) in the BWB plethysmograph. Cats then underwent either a single $5 \mathrm{~min}$ aerosol exposure with sterile saline (Pre-challenge) or successive 5 min challenges with increasing AS concentrations $(0.005 \%, 0.01 \%, 0.02 \%$, $0.04 \%$ and $0.08 \%$ ) (Challenge 1 ). The challenges of saline and AS were followed by a 5 min session of BWBP. The AS inhalations were stopped after the occurrence of a sustained dyspnoea, concurrent with Penh values exceeding $300 \%$ of post-saline baseline measurements (EAR) or after the maximum dose was reached. Respiratory parameters were assessed at $5,15,30,45,60,80,100,120,150,180,210$ and 240 min after recording the placebo challenge (Pre-challenge) or the last dose of AS (Challenge 1).

For each responsive animal (Table 1; Nos. 3, 4, 6, 7 and 8), a second AS bronchial provocation test (Challenge 2 ) was carried out by administering the same incremental doses of allergen as in Challenge 1 to test the repeatability of AS-related changes in Penh. If repeatable, the incremental doses of allergen leading up to the desired degree of airflow limitation were used for all subsequent AS-challenges (crossover trial). 
Therapeutic trial

On each study day, cats were challenged with aerosolised AS allergen. When measurements of AS-triggered EAR had been completed, the drugs were delivered to cats and each animal then tested. The functional response to inhaled bronchodilators was investigated at 5, 15, 30, 45, 60, 80, 100 and 120 min after administration. The AS bronchial provocation tests were otherwise similar in design to the initial inhalation challenge-test period (i.e. two baseline recordings with the same provocative doses of allergen and 5 min sessions of BWBP).

\section{A. suum or placebo challenges}

A. suum or saline solutions were aerosolised using an ultrasonic nebulise (Ultraneb Devilbiss 2000, Devilbiss Healthcare) known to generate particles with diameters ranging from 0.5 to $5 \mu \mathrm{m}$. The aerosol output was set at $3 \mathrm{~mL} / \mathrm{min}$. A fixed airflow through the nebuliser propelled the aerosolised particles into an animal chamber (height: $30 \mathrm{~cm}$; length: $37.5 \mathrm{~cm}$; width: $28.5 \mathrm{~cm}$ ) through a single lateral inlet ( $30 \mathrm{~mm}$ diameter). Upper outlets ( $5 \mathrm{~mm}$ diameter) allowed the safe evacuation of aerosol by an extractor hood.

\section{Drug administration}

Inhaled SAL, IB and SAL/IB were administered to AS-challenged cats using a pMDI and a spacing chamber connected to a facemask (Aerokat, Trudell Medical) immediately after recording the lung function in response to the last allergen exposure. The facemask was carefully applied on the cat's nose and mouth and the pMDI was activated once and the facemask was then held in place until the animal had taken 10 deep breaths. To avoid cross-contamination between SAL and IB, two different spacing chambers and facemasks were used. For the combination SAL/IB, the cats took 10 deep breaths before the second drug was delivered. Using a 3:2 allocation ratio, cats were randomised to one of the two different treatment sequences (sequence 1: SAL/IB; sequence 2: IB/SAL). Compliance with inhalation therapy was assessed by counting the number of failed and successful drug administrations on the basis of previous delivery procedures.

\section{Barometric whole-body plethysmography}

Data acquisition of respiratory parameters was conducted using BWBP for unrestrained conscious animals. The system adapted for cats was as previously described (Hoffman et al., 1999; Hirt et al., 2003; Kirschvink et al., 2006). Briefly, the cats were placed in the transparent Plexiglas main chamber of the BWB plethysmograph (Buxco Electronics, height: $25 \mathrm{~cm}$; length: $51 \mathrm{~cm}$; width: $30 \mathrm{~cm}$ ), ventilated by a continuous bias flow (Air Control Industries) adjusted to a flow rate of $4 \mathrm{~L} / \mathrm{min}$ by a flow meter (Influx, Cache Instrumentation). The inlet, through a screen pneumotachograph (35 mm diameter), and outlet for air were at the opposite ends of the chamber. Two sensor probes within the animal chamber continuously monitored the temperature and humidity. A differential pressure transducer (Buxco Electronics) sampled pressure signals at $100 \mathrm{~Hz}$, which were analysed on a breath-by-breath basis by a BioSystem XA software (Buxco Electronics).

The following respiratory variables were recorded: respiratory frequency (f) (cycles/min), inspiratory and expiratory times ( $\mathrm{Ti}$ and $\mathrm{Te}$ ) (ms), relaxation time (RT, i.e. the time point when $65 \%$ of tidal volume is expired) (ms), peak inspiratory and expiratory pseudoflows (PIF and PEF) $(\mathrm{mL} / \mathrm{s})$, estimated tidal volume (VT) (mL) and estimated minute volume (MV: tidal volume $\times$ respiratory frequency) $(\mathrm{mL})$. The enhanced pause was calculated using the formula: Penh $=((\mathrm{Te}-\mathrm{RT}) /$ $\mathrm{RT} \times \mathrm{PEF} / \mathrm{PIF})$. Waveforms disturbed by sniffing, vocalisations and body movements were automatically excluded by the software when VT $<10 \mathrm{~mL}, \mathrm{Ti}<150 \mathrm{~ms}, \mathrm{Ti}>10$ $000 \mathrm{~ms}$ or when the difference between the inspiratory and expiratory volumes was $>20 \%$.

Data analyses

The primary outcome was the functional response (evaluated using Penh) to AS exposure and inhaled medications. Data were expressed as the arithmetic mean \pm standard error of the mean (SEM). Statistic analysis system (SAS 9.1, SAS Institute) was used for the statistical analysis. For all analyses, differences were considered as significant when $P<0.05$.

For the initial inhalation challenge-test period, absolute Penh values were analysed by a mixed linear model to assess challenge effects (Pre-challenge, Challenges 1 and 2), changes over time and challenge by time interaction. If a significant challenge by time interaction was detected, further analyses for the individual challenges and time points were carried out.

For the therapeutic trial, Penh data were transformed before subsequent calculations and statistical analyses, and were expressed as the per cent ratio of the difference between the post-saline $(\mathrm{NaCl})$ and bronchodilator treatment (time $x$ ) values to the difference between the post-saline and EAR trigger values:

$\% \Delta$ Penh $=\frac{\text { Penh }(\text { at time } x)-\text { Penh }(\text { following } \mathrm{NaCl})}{\text { Penh }(\text { at EAR })-\text { Penh }(\text { following } \mathrm{NaCl})} \times 100$
The response to inhaled bronchodilators was first evaluated by the area under the time-response curve from 0 min (i.e. the time at which cats were treated) to $60 \mathrm{~min}\left(\mathrm{AUC}_{0-60}\right)$ and $120 \mathrm{~min}\left(\mathrm{AUC}_{0-120}\right)$ following drug administration. Both $\mathrm{AUC}_{0-60}$ and $\mathrm{AUC}_{0-120}$ were computed by trapezoidal integration. The AUC approach is a useful means to assess the overall effect of inhaled medications over a predetermined period of time (or several periods of time). Then, the times for studied parameters to achieve exactly $50 \%\left(T_{50 \%}\right)$ and $20 \%\left(T_{20 \%}\right)$ of the post-challenge values (at EAR) were calculated by linear interpolation of the time-response curves after log-transformation of time data. The durations required for half-recovery $\left(T_{50 \%}\right)$ or for returning to nearly basal conditions $\left(T_{20 \%}\right)$ provide additional information regarding whether spontaneous resolution from EAR might be accelerated by the interventional administration of an inhaled bronchodilator. $\mathrm{AUC}_{0-60}, \mathrm{AUC}_{0-120}$, $T_{20 \%}$ and $T_{50 \%}$ values were compared between the treatment groups by one-way repeated measures ANOVA with post-hoc Fischer's least significant difference tests.

\section{Results}

\section{Initial inhalation challenge-test period}

Of the eight subjects recruited in the initial inhalation challenge-test period, three were excluded from data analysis (Table 1 ; Nos. 1, 2 and 5) because they did not develop an EAR after stimulation with five doubling concentrations of the AS allergen (Fig. 2). Allergen related-changes in Penh of responsive cats are depicted in Fig. 3.

Over the $4 \mathrm{~h}$ recording period, significant changes in Penh were found between the Pre-challenge and Challenges 1 and 2 groups. A significant challenge by time interaction $(P<0.0001)$ was detected, suggesting that responses to the placebo and AS-challenges differed over time. For the Pre-challenge group, pair-wise comparisons between post-saline and any other individual time points were not significant. For both Challenges 1 and 2 groups, Penh was maximal after initiation of the allergen-induced EAR, and then gradually resumed its respective post-saline values. Penh measurements recorded after occurrence of the EAR significantly exceeded the post-saline values up to time point $60 \mathrm{~min}$. Clinically, AS-stimulated cats exhibited moderate to severe signs compatible with bronchoconstriction, such as tachypnoea ( $\sim 70$ cycles/min), expiratory dyspnoea, sporadic cough, increased and/or abnormal breath sounds on lung auscultation (wheezes, crackles) and sometimes reluctance to move. These clinical signs broadly paralleled the time course of changes in lung function. At Challenge 1, two cats (Table 1; Nos. 3 and 4) experienced an AS-induced LAR that was evidenced by a significant increase in Penh at 240 min and accompanying clinical signs of tachypnoea, moderate dyspnoea as well as increased breath sounds and low-pitched wheezes on lung auscultation.

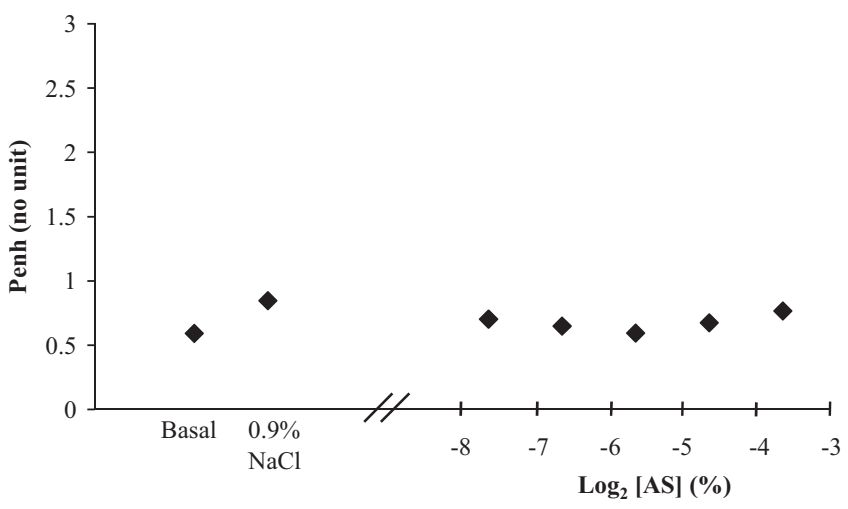

Fig. 2. Penh values recorded in an unresponsive cat (No. 2) after two baseline recording periods and successive challenges with doubling concentrations of Ascaris suum allergen. Penh, enhanced pause; Basal and $0.9 \% \mathrm{NaCl}$, Penh values recorded, respectively, at rest and following 1 min nebulisation with sterile saline; $\log _{2}$ (AS): $\log _{2}$-transformed concentrations of aerosolised A. suum allergen. 


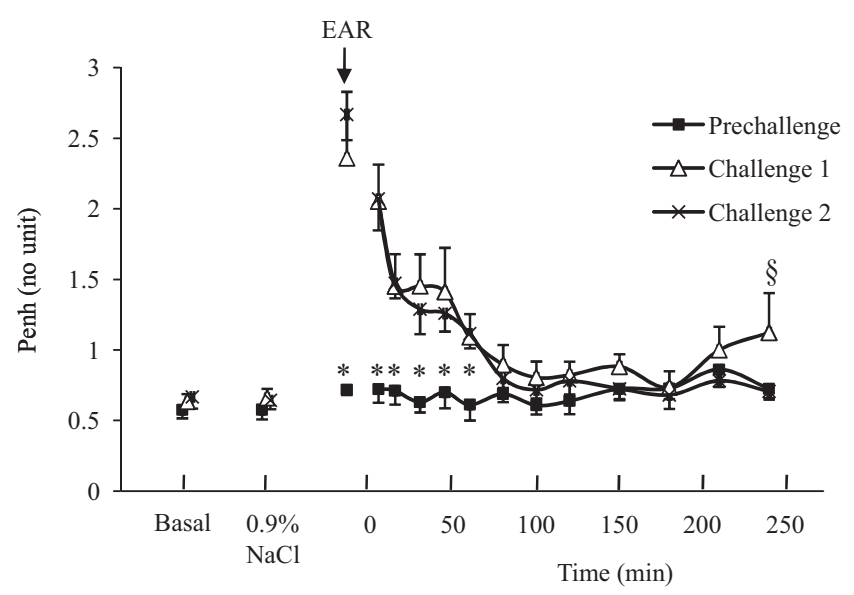

Fig. 3. Time course of mean values of Penh obtained in Ascaris suum-sensitised cats at baseline (Basal), after 1 min saline nebulisation $(0.9 \% \mathrm{NaCl})$ and following a single challenge with a placebo (Pre-challenge) or two successive challenges with $A$. suum allergen (Challenges 1 and 2) ( $n=5)$. EAR, allergen-triggered early asthmatic reaction. Data shown are the mean values \pm SEM. "Significantly different from Challenges 1 and 2, $P<0.05$. 'Significantly different from Pre-challenge and Challenge $2, P<0.05$.

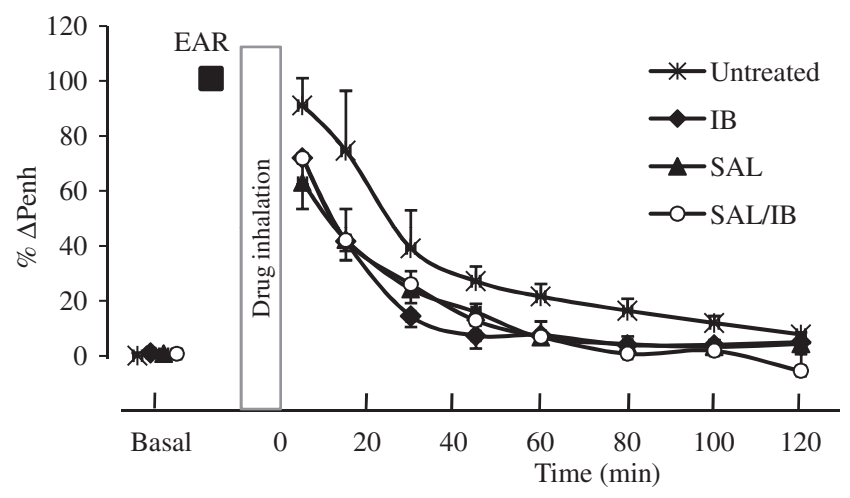

Fig. 4. Time course of mean values of $\% \Delta$ Penh obtained in Ascaris suum-sensitised cats at baseline (Basal), after allergen-triggered early asthmatic reaction (EAR) and $5,15,30,45,60,80,100$ and 120 min following inhalation of salbutamol $(100 \mu \mathrm{g})$, ipratropium bromide $(20 \mu \mathrm{g})$ or a combination of both $(100 \mu \mathrm{g} / 20 \mu \mathrm{g})$ through a pressurised metered-dose inhaler $(n=5)$. Penh data are expressed as per cent ratio of the difference between the post-saline $(\mathrm{NaCl})$ and bronchodilator treatment (time $x$ ) values to the difference between the post-saline and EAR trigger values (\% $\Delta$ Penh), and are shown as mean values with SEM.

When evaluating individual time points, pair-wise comparisons between challenges did not reveal any significant differences at rest (basal) and following $1 \mathrm{~min}$ saline nebulisation $(0.9 \% \mathrm{NaCl})$. Compared to Pre-challenge, Penh values assessed during Challenges 1 and 2 were significantly increased from the EAR trigger (EAR) up to $60 \mathrm{~min}$ but they did not differ among themselves. The LAR reported during Challenge 1 led to significant increases in Penh values at $240 \mathrm{~min}$ as compared with the Pre-challenge and Challenge 2 groups.

Considering the spontaneous recovery from EAR within 80$120 \mathrm{~min}$ and the confounding effects of inconsistent LAR, the measurement period was shortened to 120 min for the therapeutic trial.

\section{Therapeutic trial}

No adverse reactions were reported after bronchodilator intake. Compliance, previously defined as the percentage of successful bronchodilator intake, was $>93 \%$ at the end of the study. Indeed, one cat did not complete more than four deep breaths following
SAL administration, but data from this subject were not censored. Preliminary statistical analysis on the absolute Penh values obtained at baseline and after saline nebulisation did not show any significant differences among all four treatment groups (SAL, IB, SAL/IB, untreated). Notably, the functional response to allergen exposure (at EAR) was of similar degree among the four study groups $(P>0.05)$.

Time-related bronchodilating effects of SAL (100 $\mu \mathrm{g})$, IB $(20 \mu \mathrm{g})$ and SAL/IB $(100 \mu \mathrm{g} / 20 \mu \mathrm{g})$ are depicted in Fig. 4. No significant differences were found among the four study groups, with reference to the mean $A_{U C} C_{0-60}, T_{20 \%}$ and $T_{50 \%}$ values of $\% \Delta$ Penh. Mean AUC $_{0-120}$ values of $\% \Delta$ Penh were similar between the tested bronchodilators, but significantly lower than those calculated in the untreated group (Table 2).

\section{Discussion}

There is increasing recognition that feline asthma models are needed to properly assess novel therapies for the clinical disease. However, little is known whether successive allergen challenges induce repeatable changes in lung function. In this study, we first confirmed the reproducibility of two consecutive allergenic stimulations as a prerequisite for the subsequent evaluation of bronchodilators according to a crossover design. Mainstay therapy for feline asthma traditionally relies on corticosteroids to dampen the underlying airway inflammation and on bronchodilators to counter the acute airflow limitation. Although bronchodilators delivered by pMDI are empirically used in feline asthmatic patients, their efficacy, duration and onset of action, in addition to their optimal dosage, have yet to be quantitatively assessed. The technique of BWBP was used to address some of these issues in the current study. Under the experimental conditions, neither the single-drug treatments (SAL, IB) nor the combined medication (SAL/IB) significantly reduced the times for recovery $\left(T_{50 \%}, T_{20 \%}\right)$ and the allergen-elicited changes in Penh averaged over the first 60 min after administration $\left(\mathrm{AUC}_{0-60}\right)$. Among the Penh-derived parameters, SAL and IB as monotherapies significantly affected AUC $_{0-120}$, without additional benefit when combining them.

Two successive allergen provocation tests carried out on actively sensitised cats triggered repeatable EAR peaking within the first minute after AS exposure, which spontaneously resolved 80 min later. Consistent with these findings, a substantial increase in lung resistance was previously recorded in anesthetised AS-sensitised cats within $10 \mathrm{~min}$ following acute allergen exposure, but was not observed 90 min later (Padrid et al., 1995). Not only has the feline asthma model based on AS sensitisation successfully mimicked a fundamental feature of the asthma phenotype, namely an immediate and reversible bronchoconstriction after allergen exposure, but it has also pinpointed the progressive time-related recovery from EAR within the current experimental framework. In this context, computing the AUC of the plotted data against time is a simple and valuable method to correct for this unavoidable confounding factor when assessing bronchodilator efficacy. Viewed from a clinical perspective, the spontaneous resolution from EAR raises concerns about how useful inhaled bronchodilators would be in relieving acute bronchospasm in cats with naturally developing asthma. Therefore, attempts were made to assess whether this spontaneous resolution rate might be accelerated by the interventional administration of an inhaled bronchodilator. Determining the delay after which time cats with allergen-induced bronchoconstriction return to nearly basal conditions $\left(T_{20 \%}\right)$ is a complementary approach to AUC calculations for evaluating bronchodilator effects, while accounting for the bias due to spontaneous recovery. 
Table 2

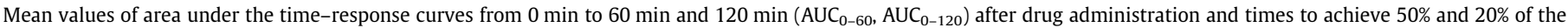

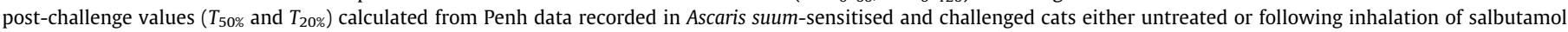
$(100 \mu \mathrm{g})$, ipratropium bromide $(20 \mu \mathrm{g})$ or a combination of both $(100 \mu \mathrm{g} / 20 \mu \mathrm{g})$ through a pressurised metered-dose inhaler $(n=5)$.

\begin{tabular}{|c|c|c|c|c|c|}
\hline Parameters & Untreated & SAL through pMDI $(100 \mu \mathrm{g})$ & IB through pMDI $(20 \mu \mathrm{g})$ & SAL/IB through pMDI $(100 \mu \mathrm{g} / 20 \mu \mathrm{g})$ & $P$ \\
\hline \multicolumn{6}{|l|}{$\% \Delta$ Penh } \\
\hline $\mathrm{AUC}_{0-60}$ & $3021 \pm 638$ & $1904 \pm 235$ & $1693 \pm 172$ & $1949 \pm 638$ & 0.063 \\
\hline $\mathrm{AUC}_{0-120}$ & $3879 \pm 817^{a}$ & $2164 \pm 228^{b}$ & $1974 \pm 324^{\mathrm{b}}$ & $2016 \pm 817^{b}$ & 0.039 \\
\hline$T_{20 \%}(\min )$ & $63.2 \pm 9.5$ & $38.8 \pm 5.5$ & $39.1 \pm 5.4$ & $42.0 \pm 7.2$ & 0.079 \\
\hline$T_{50 \%}(\min )$ & $25.7 \pm 8.8$ & $9.5 \pm 2.9$ & $10.0 \pm 2.4$ & $11.9 \pm 2.3$ & 0.065 \\
\hline
\end{tabular}

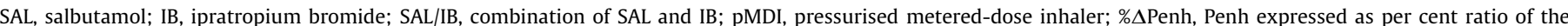

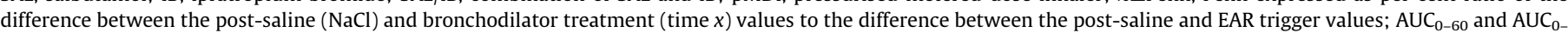

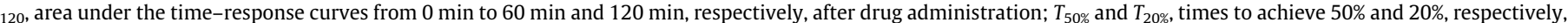
of the post-challenge values.

Data shown are the mean values \pm SEM. NS, not significant.

${ }^{\mathrm{a}, \mathrm{b}}$ Data with different superscripts are significantly different, $P<0.05$.

Successive exposures with doubling doses of allergen would be expected to trigger an EAR in the first eight recruited AS-sensitised cats. Although the number of responding animals appeared to be related to the concentrations of challenging allergen, acute bronchospasm was not observed in all the sensitised cats even at the highest concentrations (5/8 responders; 63\%). Other investigators have previously established an EAR occurrence of approximately $60 \%$ in human asthmatics undergoing an environmental challenge, during which low amounts of allergen particles dispersed in the ambient air are inhaled continuously over a long period of time (Arvidsson et al., 2007). In spite of the presumed remarkable differences in deposition of allergen within the airways, the experimental challenge undertaken in this study reflected the asthmatic response under normal environmental conditions well. Because allergen-induced eosinophilic airway inflammation has been previously demonstrated in unresponsive cats (Table 1; Nos. 1, 2 and 5), it should be borne in mind that the lack of functional evidence for an EAR does not preclude the subsequent development of a delayed pulmonary inflammatory response (LAR), which is of clinical importance. Finally, the reproducibility of LAR may be questionable, because some responsive cats (Table 1; Nos. 3 and 4) showed inconsistent LAR between Challenges 1 and 2. It is conceivable that both these cats responded well beyond the last time point assessed (240 $\mathrm{min}$ ) for Challenge 2 , paralleling the case of human beings in whom the onset of LAR is quite variable, occurring as early as $3 \mathrm{~h}$ or as late as $8 \mathrm{~h}$ after exposure to allergen (O’Byrne et al., 1987).

Neither incremental doses of inhaled allergen nor 12 consecutive challenges with fixed doses of aeroallergen (Norris Reinero et al., 2004) proved to be sufficient to elicit an EAR in research cats with experimental asthma. Thus, factors other than the intensity of allergen exposure during challenge appear to impact the occurrence and magnitude of allergen-induced EAR. The level of allergic sensitisation (i.e. the circulating level of allergen-specific IgE) and the degree of underlying bronchial hyperresponsiveness to nonallergic stimuli, such as histamine and methacholine, are believed to be crucial determinants of airway responsiveness to allergen (Cockcroft and Davis, 2006).

Airway hyperresponsiveness can be defined as an increase in the ease and degree of airway narrowing in response to bronchoconstrictor stimuli in vivo. According to Alvarez-Puebla et al. (2001), the development of an EAR in asthmatic patients is possibly related to the degree of baseline bronchial hyperresponsiveness to methacholine. The higher the provocative dose of methacholine that produced $>20 \%$ decrease in forced expiratory volume in $1 \mathrm{~s}$ below the post-diluent value $\left(\mathrm{PD}_{20} \mathrm{FEV}_{1}\right)$, the higher was the threshold dose of allergen required to achieve the same effect. In the present study, airway responsiveness to inhaled carbachol was assessed by BWBP before entry into the initial inhalation challenge-test period, but no relationship was found between provocative doses of allergen and carbachol (data not shown), which suggested that the degree of baseline bronchial responsiveness as measured by carbachol challenge was not expected to be a contributory factor towards the airway response to allergen in the present model.

In humans, the degree of cutaneous allergic reactivity to allergen, evaluated by the skin-prick test, is known to correlate well with circulating levels of allergen-specific IgE in the serum, and these levels have shown high capability to predict airway responsiveness to allergen (Bryant et al., 1975; Bryant and Burns, 1976). In this study, we did not determine the degree of allergic sensitisation (evaluated by intradermal skin testing in small animals or by serum-specific IgE levels), although this could have been helpful to distinguish the high- from low-responders among the AS-sensitised cats.

The application of BWBP as a non-invasive pulmonary function test and the use of its derived parameter, Penh, as a surrogate for conventional measurements (i.e. respiratory mechanics) of airway resistance have been severely criticised (Mitzner and Tankersley, 2003; Bates et al., 2004; Adler et al., 2004; Lundblad et al., 2007). The pressure signal from the breathing of an animal in the plethysmographic box reflects both the effects of gas conditioning (heating and humidification during inspiration versus cooling and drying during expiration) and the effects of airway resistance on alveolar pressure gradients that promote gas expansion during inspiration and compression during expiration. According to some investigators, pressure changes in a single-chamber BWB plethysmograph are essentially dependent upon gas conditioning and therefore, Penh, which is based on the box pressure during expiration, cannot be assumed as a reliable estimator of bronchoconstriction but, at best, as a function of the ventilatory timing (e.g. respiratory frequency) (Mitzner and Tankersley, 2003; Schwarze et al., 2005: author reply). In the present study, no significant correlations $(P>0.05)$ were found between Penh and respiratory rate (data not shown), in agreement with earlier experiments performed in mice (Hamelmann et al., 1997). In contrast, clinical signs compatible with bronchoconstriction (expiratory dyspnoea, coughing and wheezes) broadly paralleled the time course of Penh. Although the validity of Penh as a surrogate for lung resistance has still to be proven in cats under varying respiratory conditions, these findings along with others (Rozanski and Hoffman, 1999) tended to indicate that the parameter Penh was well suited to assess airflow limitation in cats with experimentally induced or naturally occurring bronchospasm.

We believe this is the first study to examine SAL, IB and the combination SAL/IB delivered through pMDI for reversing allergen-induced bronchospasm in cats. Neither bronchodilator substantially improved lung function, as evidenced by $\mathrm{AUC}_{0-60}$, 
$T_{50 \%}$ and $T_{20 \%}$. Although the tested medications significantly alleviated AS-elicited changes in Penh averaged over the 120 min observation period $\left(A_{U C} C_{0-120}\right)$, no such effects were noted over the first $60 \mathrm{~min}\left(\mathrm{AUC}_{0-60}\right)$, when clinical status and lung function are supposed to be most altered (see Initial inhalation challenge-test period). This may be unsatisfactory from a clinical standpoint. In line with these negative findings are the observations in the recent literature that regular nebulisation of SAL in experimentally asthmatic cats did not lessen the severity of symptoms the animals displayed immediately after delivery of allergen (Reinero et al., 2009).

Our results contrast sharply with those of a previous study conducted by our group, in which inhalation of either SAL, IB or their synergistic combination successfully prevented carbachol-induced bronchoconstriction in healthy cats (Leemans et al., 2009). It is conceivable that discrepancies between both studies may be related to methodological issues for the following reasons: Firstly, the fact that inhalation of the bronchodilators was preventive in the former study and curative in the latter could suggest differential drug exposure. Considering the approach implemented in the current study, AS-related changes in airway calibre were thought to reduce aerosol lung deposition, thereby increasing the likelihood of poor response to inhaled bronchodilators. Secondly, carbachol has a bronchoconstrictor effect by acting directly on the airway smooth muscle, whereas an allergen is an indirect stimulus acting through the release of a number of mediators, which may cause airway narrowing by mechanisms other than by airway smooth-muscle contraction. Presumably the cats showed persistent airway obstruction in spite of inhaling bronchodilators because of moderate to severe bronchial wall oedema and possibly airway mucus plugging.

The inhaled bronchodilators tested in this study did not affect measures of airflow limitation as much as expected from the results of preliminary clinical studies using BWBP as pulmonary function testing (Rozanski and Hoffman, 1999; Hirt and Dederichs, 2001). In cats with diagnosed asthma, BWBP derived parameters (Penh, Pause) effectively quantified both lower airway obstruction and its reversal by treatment with SAL through pMDI (Rozanski and Hoffman, 1999) or nebulised terbutaline (Hirt and Dederichs, 2001). However, there were no untreated asthmatic cats as controls and no details on standardised intervals between drug administration and plethysmographic records, so these studies may have missed the spontaneous recovery from the acute respiratory distress, which was critical in our model of allergic asthma. Therefore, any comparisons with clinical data should be interpreted with caution until randomised controlled clinical trials are performed in pet cats with asthma.

Administration of unsuitable drug doses may also have contributed to the apparent lack of activity of inhaled bronchodilators, particularly when the airways are obstructed since penetration of inhaled particles will be impaired. In this study, the choice of drug dosage was based on recent recommendations for the prophylactic use of inhaled bronchodilators in cats (Leemans et al., 2009). Therefore, doses of bronchodilators higher than those recommended may be needed to achieve effects reported from previous pre-clinical and clinical trials (Rozanski and Hoffman, 1999; Hirt and Dederichs, 2001; Leemans et al., 2009). It may be that between-group variability in AS-elicited changes in Penh led to biased estimation of bronchodilators' efficacy. Nevertheless, statistical analyses did not detect any significant differences between study groups (untreated, SAL, IB, SAL/IB) for response to allergenic stimulation. This may be due to the study being underpowered $(P<0.8)$, particularly when considering the small numbers of experimental subjects. It should also be noted that a sustained bronchoconstriction would have permitted more accurate assessment of drug efficacy in the present model.

\section{Conclusions}

Established on the basis of AS sensitisation and inhalation, the current feline model of allergen-induced bronchoconstriction offers a standardised and repeatable approach for assessment of bronchodilating drugs. Unexpectedly, inhaled SAL and IB, used as single agents or for their combined therapeutic effect, did not substantially reverse the allergen-induced bronchospasms in AS-sensitised cats. Research on cats with experimentally induced or naturally occurring bronchospasm on a larger scale is required to further explore ideal dosages and optimal dosing frequency of currently available inhaled bronchodilators or to look for more effective ways to administer suitable drugs to the respiratory system.

\section{Conflict of interest statement}

None of the authors of this paper has a financial or personal relationship with other people or organisations that could inappropriately influence or bias the content of the paper.

\section{Acknowledgements}

This study was carried out at the Department for Functional Sciences of the Faculty of Veterinary Medicine, University of Liège, Belgium. Jérôme Leemans is a graduate student supported by FRIA, Belgium. This study was supported by a grant from the Région Wallonne DGTRE, Belgium. The authors are grateful to I. Habsch for her technical assistance.

\section{References}

Adler, A., Cieslewicz, G., Irvin, C.G., 2004. Unrestrained plethysmography is an unreliable measure of airway responsiveness in BALB/C and C57BL/6 mice. Journal of Applied Physiology 97, 286-292.

Alvarez-Puebla, M.J., Olaguibel-Rivera, J.M., Urbiola-Marcilla, E., Garcia, B.E., TabarPurroy, A.I., 2001. Determinants of allergen-induced late bronchial responses in mild asthmatics. Chest 119, 120-127.

Arvidsson, M.B., Lowhagen, O., Rak, S., 2007. Early and late phase asthmatic response in lower airways of cat-allergic asthmatic patients - a comparison between experimental and environmental allergen challenge. Allergy 62, 488494.

Bates, J., Irvin, C., Brusasco, V., Drazen, J., Fredberg, J., Loring, S., Eidelman, D., Ludwig, M., Macklem, P., Martin, J., Milic-Emili, J., Hantos, Z., Hyatt, R., Lai-Fook, S., Leff, A., Solway, J., Lutchen, K., Suki, B., Mitzner, W., Pare, P., Pride, N., Sly, P., 2004. The use and misuse of Penh in animal models of lung disease. American Journal of Respiratory Cell and Molecular Biology 31, 373-374.

Boothe, D.M., 2004. Drugs affecting the respiratory system. In: King, L.G. (Ed.), Respiratory Disease in Dogs and Cats. Saunders, St. Louis, Missouri, USA, pp 229-252.

Bousquet, J., Jeffery, P.K., Busse, W.W., Johnson, M., Vignola, A.M., 2000. Asthma from bronchoconstriction to airways inflammation and remodeling. American Journal of Respiratory and Critical Care Medicine 161, 1720-1745.

Bryant, D.H., Burns, M.W., 1976. Bronchial histamine reactivity: its relationship to the reactivity of the bronchi to allergens. Clinical Allergy 6, 523-532.

Bryant, D.H., Burns, M.W., Lazarus, L., 1975. The correlation between skin tests, bronchial provocation tests and the serum level of IgE specific for common allergens in patients with asthma. Clinical Allergy 5, 145-157.

Cockcroft, D.W., Davis, B.E., 2006. Airway hyperresponsiveness as a determinant of the early asthmatic response to inhaled allergen. Journal of Asthma 43, 175178.

Halloy, D.J., Kirschvink, N.A., Vincke, G.L., Hamoir, J.N., Delvaux, F.H., Gustin, P.G., 2004. Whole body barometric plethysmography: a screening method to investigate airway reactivity and acute lung injuries in freely moving pigs. The Veterinary Journal 168, 276-284.

Hamelmann, E., Schwarze, J., Takeda, K., Oshiba, A., Larsen, G.L., Irvin, C.G., Gelfand, E.W., 1997. Noninvasive measurement of airway responsiveness in allergic mice using barometric plethysmography. American Journal of Respiratory and Critical Care Medicine 156, 766-775.

Hawkins, E.C., Kennedy-Stoskopf, S., Levy, J., Meuten, D.J., Cullins, L., DeNicola, D., Tompkins, W.A. Tompkins, M.B., 1994. Cytologic characterization of bronchoalveolar lavage fluid collected through an endotracheal tube in cats. American Journal of Veterinary Research 55, 795-802.

Hirt, R.A., 2005. Feline asthma - a review and new insights. European Journal of Companion Animal Practice 15, 141-154.

Hirt, R.A., Dederichs, D., 2001. Quantitation of bronchoconstriction and response to nebulized beta2-bronchodilators in spontaneously occurring feline asthma. In: 
Proceedings of the 7th Annual Meeting of the Federation of European Companion Animal Veterinary Associations, Berlin, Germany, pp. 41-42.

Hirt, R.A., Dederichs, D., Boehler, A., Hoffman, A.M., 2003. Relationship of age, sex, body weight, and hematologic and respiratory variables with airway reactivity in adult cats. American Journal of Veterinary Research 64, 26-31.

Hirt, R.A., Leinker, S., Mosing, M., Wiederstein, I., 2008. Comparison of barometric whole body plethysmography and its derived parameter enhanced pause (PENH) with conventional respiratory mechanics in healthy Beagle dogs. The Veterinary Journal 176, 232-239.

Hoffman, A.M., Dhupa, N., Cimetti, L., 1999. Airway reactivity measured by barometric whole-body plethysmography in healthy cats. American Journal of Veterinary Research 60, 1487-1492.

Kirschvink, N., Leemans, J., Delvaux, F., Snaps, F., Clercx, C., Gustin, P., 2007. Functional, inflammatory and morphological characterisation of a cat model of allergic airway inflammation. The Veterinary Journal 174, 541-553.

Kirschvink, N., Leemans, J., Delvaux, F., Snaps, F., Marlin, D., Sparkes, A., Clercx, C., Gustin, P., 2006. Non-invasive assessment of growth, gender and time of day related changes of respiratory pattern in healthy cats by use of barometric whole body plethysmography. The Veterinary Journal 172, 446454.

Kirschvink, N., Vincke, G., Onclinx, C., Peck, M.J., Gustin, P., 2005. Comparison between pulmonary resistance and Penh in anaesthetised rats with tracheal diameter reduction and after carbachol inhalation. Journal of Pharmacologica and Toxicological Methods 51, 123-128.

Lee-Fowler, T.M., Cohn, L.A., Declue, A.E., Spinka, C.M., Reinero, C.R., 2009 Evaluation of subcutaneous versus mucosal (intranasal) allergen-specific rush immunotherapy in experimental feline asthma. Veterinary Immunology and Immunopathology 129, 49-56.

Leemans, J., Kirschvink, N., Bernaerts, F., Clercx, C., Cambier, C., Gustin, P., 2009. A pilot study comparing the antispasmodic effects of inhaled salmeterol, salbutamol and ipratropium bromide using different aerosol devices on muscarinic bronchoconstriction in healthy cats. The Veterinary Journal 180, 236-245.

Leemans, J., Kirschvink, N., Cambier, C., Clercx, C., Gustin, P., 2008. Oral and inhaled corticosteroids decrease eosinophilic airway inflammation and bronchial reactivity in Ascaris suum-sensitized and challenged cats. In: Proceedings of the 26th Symposium of the Veterinary Comparative Respiratory Society, Oklahoma City, OK, USA

Lundblad, L.K., Irvin, C.G., Hantos, Z., Sly, P., Mitzner, W., Bates, J.H., 2007. Penh is not a measure of airway resistance! European Respiratory Journal 30, 805.

Mitzner, W., Tankersley, C., 2003. Interpreting Penh in mice. Journal of Applied Physiology 94, 828-831.
Norris Reinero, C.R., Decile, K.C., Berghaus, R.D., Williams, K.J., Leutenegger, C.M., Walby, W.F., Schelegle, E.S., Hyde, D.M., Gershwin, L.J., 2004. An experimental model of allergic asthma in cats sensitized to house dust mite or Bermuda grass allergen. International Archives of Allergy and Immunology 135, 117-131.

O'Byrne, P.M., Dolovich, J., Hargreave, F.E., 1987. Late asthmatic responses. American Review of Respiratory Disease 136, 740-751.

Padrid, P., 2000. Feline asthma: diagnosis and treatment. Veterinary Clinics of North America: Small Animal Practice 30, 1279-1293.

Padrid, P., Snook, S., Finucane, T., Shiue, P., Cozzi, P., Solway, J., Leff, A.R., 1995. Persistent airway hyperresponsiveness and histologic alterations after chronic antigen challenge in cats. American Journal of Respiratory and Critical Care Medicine 151, 184-193.

Padrid, P.A., Cozzi, P., Leff, A.R., 1996. Cyclosporine A inhibits airway reactivity and remodeling after chronic antigen challenge in cats. American Journal of Respiratory and Critical Care Medicine 154, 1812-1818.

Padrid, P.A., Feldman, B.F., Funk, K., Samitz, E.M., Reil, D., Cross, C.E., 1991. Cytologic microbiologic, and biochemical analysis of bronchoalveolar lavage fluid obtained from 24 healthy cats. American Journal of Veterinary Research 52, 1300-1307.

Reinero, C.R., Byerly, J.R., Berghaus, R.D., Berghaus, L.J., Schelegle, E.S., Hyde, D.M., Gershwin, L.J., 2006. Rush immunotherapy in an experimental model of feline allergic asthma. Veterinary Immunology and Immunopathology 110, 141-153.

Reinero, C.R., Cohn, L.A., Delgado, C., Spinka, C.M., Schooley, E.K., Declue, A.E. 2008 Adjuvanted rush immunotherapy using $\mathrm{CpG}$ oligodeoxynucleotides in experimental feline allergic asthma. Veterinary Immunology and Immunopathology 121, 241-250.

Reinero, C.R., Decile, K.C., Byerly, J.R., Berghaus, R.D., Walby, W.E., Berghaus, L.J., Hyde, D.M., Schelegle, E.S., Gershwin, L.J., 2005. Effects of drug treatment on inflammation and hyperreactivity of airways and on immune variables in cats with experimentally induced asthma. American Journal of Veterinary Research $66,1121-1127$.

Reinero, C.R., Delgado, C., Spinka, C., Declue, A.E., Dhand, R., 2009. Enantiomerspecific effects of albuterol on airway inflammation in healthy and asthmatic cats. International Archives of Allergy and Immunology 150, 43-50.

Rozanski, E.A., Hoffman, A.M., 1999. Lung function and inhaled albuterol in cats with asthma. Journal of Veterinary Internal Medicine 13, 259.

Schooley, E.K., Gee Turner, J.B., Jiji, R.D., Spinka, C.M., Reinero, C.R., 2007. Effects of cyproheptadine and cetirizine on eosinophilic airway inflammation in cats with experimentally induced asthma. American Journal of Veterinary Research 68, 1265-1271.

Schwarze, J., Hamelmann, E., Gelfand, E.W., 2005. Barometric whole body plethysmography in mice. Journal of Applied Physiology 98, 1955-1957. 